\title{
Granuloma Telangiectásico y Pérdida Ósea asociado a tratamiento Anovulatorio
}

Odontólogo. Magíster en Salud Pública. Profesor Auxiliar, Facultad de Odontología, Universidad de Cartagena (Colombia). Director del grupo de investigación "GISPOUC".

b Odontólogo. Especialista en Periodoncia. Magíster en Educación. Docente titular, Universidad de Cartagena (Colombia). Director Grupo de Investigación "GITOUC".

c Investigadora de la Facultad de Odontología, Universidad de Cartagena (Colombia).

Correspondencia: Antonio Díaz Caballero, Facultad de Odontología, Universidad de Cartagena. Campus de la Salud, Barrio Zaragocilla. Cartagena, Colombia. Correo electrónico: adiazc1@unicartagena.edu.co

Recibido el 28 de octubre 2010. Aceptado para su publicación el 16 de diciembre de 2010.
Farith González Martínez ${ }^{\mathrm{a}}$, Antonio Díaz Caballerob ${ }^{\mathrm{b}}$ Zulieth López Arrietac

\section{RESUMEN}

El granuloma telangiectásico se incluye dentro de las patologías reactivas inflamatorias orales asociadas a efectos secundarios de la ingesta de anovulatorios. Sus características clínicas no son patognomónicas, por lo que su diagnosis se debe realizar con apoyo en estudios histológicos. En cuanto a su incidencia, es mayor en mujeres que se encuentran en la segunda y tercera décadas de la vida. Aunque el factor etiológico aún es discutido, existe evidencia clara de que es una reacción que conjuga la presencia de factores irritantes locales, como la biopelícula, y condiciones sistémicas, como lo son las alteraciones hormonales.

Palabras Clave. Granuloma Telangiecticum, Anticonceptivos Hormonales Orales.

\section{ABSTRACT}

Pyogenic Granuloma with Bone Loss in a patient taking Contraceptives

Pyogenic Granuloma is one of the reactive inflammatory oral diseases associated with contraceptive intake. The clinical features are not pathognomonic therefore its diagnosis must be made with histological support. Its incidence is higher in women who are in the second and third decade of life. Although its aetiology remains controversial there is clear evidence that it is a reaction to the presence of local irritants such as biofilms together with systemic conditions such as hormonal changes.

Key words. Granuloma, Pyogenic, Contraceptives, Oral, Hormonal.

\section{INTRODUCCIÓN}

El granuloma telangiectásico se describe como una patología hiperplásica reactiva inflamatoria ${ }^{1}$. Aparece como una tumoración enrojecida o púrpura, de superficie lisa o lobulada, con apariencia brillante o con ulceraciones, y presenta una tendencia hemorrágica ${ }^{2}$. El tamaño varía entre unos pocos milímetros a varios centímetros, y se presenta con mayor frecuencia en mujeres embarazadas, o que ingieren anticonceptivos orales ${ }^{3}$. El lugar predilecto de aparición es la encía ${ }^{4,5}$ y puede ocasionar molestias a la masticación, hemorragia, afección estética y trastornos periodontales ${ }^{2}$. Cuando el proceso inflamatorio del granuloma telangiectásico llega a hueso se pueden presentar reabsorciones externas ${ }^{6}$ o pérdidas óseas en forma de flecha ${ }^{2}$.

\section{OBSERVACIONES CLÍNICAS}

Paciente femenina de 39 años de edad sin alteraciones sistémicas, con cuadro hemático normal y con historia de consumo crónico de anovulatorios. Acudió a consulta por presentar inconformidad estética y funcional por lesión hiperplásica hemorrágica asociada a órgano dental antero superior derecho. Clínicamente se observó lesión eritematosa de $1,5 \mathrm{~cm}$ de diámetro que com- 
prometía todas las superficies de órgano dental 11, el cual se encontraba extruido, sintomático, con movilidad incrementada. Se detectó bolsa periodontal al sondeo de $7 \mathrm{~mm}$ y se percibió material calcáreo adscrito a la zona radicular. Radiográficamente se observó pérdida ósea vertical coherente con la localización de la hiperplasia. El examen histopatológico reveló alteración formada por proliferación fibricolágena con vascularización aumentada e infiltrado inflamatorio crónico compatible con el diagnóstico de granuloma telangiectásico. Anterior a la intervención quirúrgica se realizó fase etiotrópica periodontal.

La fase quirúrgica se inició con la infiltración anestésica de la zona con lidocaína al $2 \%$. A continuación se procedió a realizar escisión de la hiperplasia con bisturí manual. El corte se realizó en tejido sano a $1 \mathrm{~mm}$ de la hiperplasia propiamente dicha, desbridando hasta exponer periostio, el cual fue cureteado. La exodoncia del órgano dental 11 se inició con sindesmotomía, luego se continuó con la luxación y por último la tracción del diente con ayuda de un fórceps 150. El campo operatorio fue detoxificado con tetraciclina. La sutura se realizó con técnica en asa en ocho y para este procedimiento se utilizó seda negra trenzada 4-0. La zona intervenida y el alveolo se topicó con Cloruro Férrico como medio astringente que permitió obtener mayor hemostasia. Se realizó evaluación clínica a los ocho días para retirar las suturas.

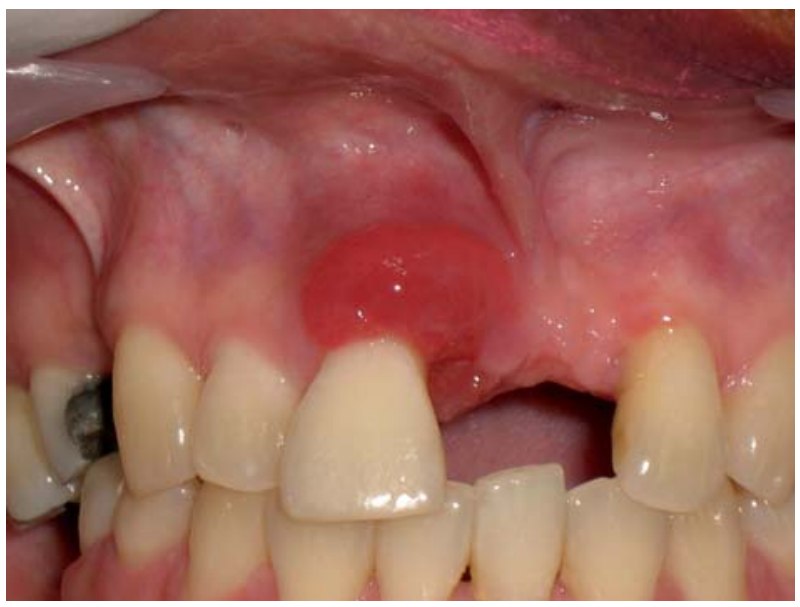

Figura 1. Lesión hiperplásica eritematosa de gran tamaño circunscrita al incisivo central superior derecho.

\section{COMENTARIOS}

El reto para realizar un acierto diagnostico en el granuloma telangiectásico radica en la deficiencia de aportes clínicos patognomónicos, por lo que la diagnosis definitiva se obtiene con un estudio histopatológico, el cual se caracteriza por la presencia de proliferación fibrovascular benigna ${ }^{3}$, además se evidencian células polimorfonucleares y células inflamatorias crónicas que se localizan frecuentemente en todo el estroma edematoso con la formación de un micro absceso. Los fibroblastos son de mayor tamaño y la actividad de reproducción celular se encuentra aumentada. A diferencia en las lesiones seniles, se aprecian prevalentemente los fibrocitos y hay un predominio de células plasmáticas ${ }^{3,7}$.

Los trastornos endocrinos ocasionados por el embarazo o la ingesta de anovulatorios ocasionan fisiológicamente modificaciones en la función y organización arquitectural de la sangre y la linfa. El estrógeno es capaz de estimular los factores productores de macrófagos, granulocitos, queratinocitos, fibroblastos y factor de crecimiento transformante beta 1, por lo que se infiere que induce a la formación de tejido de granulación ${ }^{7}$. Estas condiciones sistémicas sumadas al factor irritante o traumático crónico local, como es la placa dental, el cálculo y el trauma oclusal, inducen a la aparición del granuloma telangiectásico ${ }^{3,5}$.

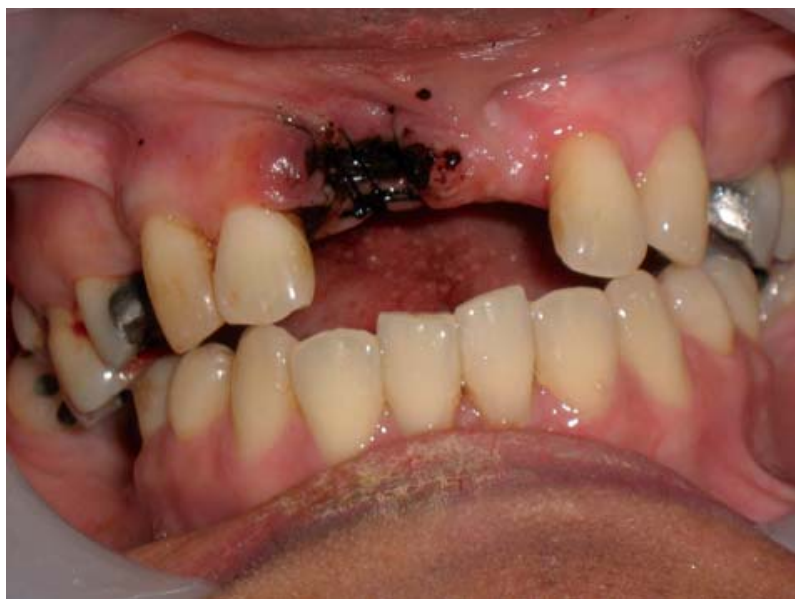

Figura 2. Campo operatorio tras la escisión quirúrgica, exodoncia, medicación con tetraciclina y cloruro férrico y suturas. 
El tratamiento del granuloma telangiectásico se reduce a una escisión quirúrgica del tejido granular con curetaje minucioso hasta el periostio, lo que nos asegura evitar una recidiva ${ }^{2,5}$. Puede realizarse con bisturí, con electrobisturí, con láser o criocirugía, y el uso de corticoides también se implementa en algunas oportunidades ${ }^{7,8}$.

El granuloma telangiectásico es una patología oral inducida por el consumo de anovulatorios, por lo que se considera a las mujeres que se encuentran bajo estas condiciones farmacológicas como población de alto riesgo frente a la posibilidad del desarrollo de esta hiperplasia. Sin embargo, hay que tener en cuenta que sólo la presencia del factor sistémico involucrado no es concluyente en la aparición de esta patología si no existe un factor irritante local, por lo cual la intervención odontológica temprana y frecuente que elimine y controle la biopelícula, cálculos dentales y restauraciones defectuosas se convierte en un factor protector frente a la incidencia de esta enfermedad en el grupo poblacional mencionado.

\section{BIBLIOGRAFÍA}

1. Das Chagas MS, Pinheiro Rdos S, Janini ME, Maia LC. Pyogenic granuloma: lobular capillary hemangioma in the upper lip of a 24-month-old child: case report. J Dent Child (Chic). 2009; 76(3):237-40.

2. Shenoy SS, Dinkar AD. Pyogenic granuloma associated with bone loss in an eight year old child: A case report. J Indian Soc Pedod Prev Dent. 2006; 24(4):201-3.

3. Dojcinovic I, Richter M, Lombardi T. Occurrence of a pyogenic granuloma in relation to a dental implant. J Oral Maxillofac Surg. 2010; 68(8):1874-6.

4. Al-Khateeb T, Ababneh K. Oral pyogenic granuloma in Jordanians: a retrospective analysis of 108 cases. J Oral Maxillofac Surg. 2003; 61(11):1285-8.

5. de Souza AG, da Silva BC, Israel MS, Lindenblatt R, de Andrade AM, Ramos ME. Atypical location of pyogenic granuloma in two pediatric patients. Gen Dent. 2008; 56(5):44750.

6. Vossoughi R, Takei HH. External cervical resorption associated with traumatic occlusion and pyogenic granuloma. J Can Dent Assoc. 2007; 73(7):625-8.

7. Jafarzadeh $\mathrm{H}$, Sanatkhani M, Mohtasham N. Oral pyogenic granuloma: a review.J Oral Sci. 2006; 48(4):167-75.

8. Lindenmüller IH, Noll P, Mameghani T, Walter C. CO2 laserassisted treatment of a giant pyogenic granuloma of the gingiva. Int J Dent Hyg. 2010; 8(3):249-52. 